\title{
Review \\ Current Role of Computed Tomography in the Evaluation of Acute Coronary Syndrome
}

\author{
Min Ji Son ${ }^{1}$, Seung Min Yoo ${ }^{1, *}$, Dongjun Lee ${ }^{2}{ }^{\mathbb{D}}$, Hwa Yeon Lee ${ }^{3}$, In Sup Song ${ }^{4}$, Eun Ju Chun ${ }^{5}$ \\ and Charles S White ${ }^{6}$ \\ 1 Department of Radiology, CHA University Bundang Medical Center, Bundang 13497, Korea; \\ a176008@chamc.co.kr \\ Military Service in Korean Army, Hongcheon 25117, Korea; sirious0416@naver.com \\ 3 Smile Radiologic Clinic, Seoul 33066, Korea; hynlee1@hanmail.net \\ 4 Department of Radiology, Chun Ju Jesus General Hospital, Chun Ju 54987, Korea; issong1@nate.com \\ 5 Department of Radiology, Seoul National University Bundang Medical Center, Seongnam 13620, Korea; \\ drejchun@hanmail.net \\ 6 Department of Radiology, University of Maryland, Baltimore, MD 21201, USA; cwhite@umm.edu \\ * Correspondence: smyoo@cha.ac.kr; Tel.: +82-3-780-5423
}

check for

updates

Citation: Son, M.J.; Yoo, S.M.; Lee, D.; Lee, H.Y.; Song, I.S.; Chun, E.J.; White, C.S. Current Role of Computed Tomography in the Evaluation of Acute Coronary Syndrome.

Diagnostics 2021, 11, 266. https:// doi.org/10.3390/diagnostics11020266

Academic Editor: Michael Henein

Received: 30 December 2020

Accepted: 7 February 2021

Published: 9 February 2021

Publisher's Note: MDPI stays neutral with regard to jurisdictional claims in published maps and institutional affiliations.

Copyright: (c) 2021 by the authors. Licensee MDPI, Basel, Switzerland. This article is an open access article distributed under the terms and conditions of the Creative Commons Attribution (CC BY) license (https:// creativecommons.org/licenses/by/ $4.0 /)$.
Abstract: This review article provides an overview regarding the role of computed tomography $(\mathrm{CT})$ in the evaluation of acute chest pain (ACP) in the emergency department (ED), focusing on characteristic CT findings.

Keywords: computed tomography; coronary CT angiography; acute coronary syndrome; acute chest pain

\section{Introduction}

Acute chest pain (ACP) is the second most common presentation in the emergency department (ED) [1]. As missed diagnosis of acute coronary syndrome (ACS) is frequently associated with a poor clinical outcome, immediate diagnosis and exclusion of ACS are of paramount importance. In patients with a high risk of ACS, prompt invasive coronary angiography and/or revascularization should be performed without noninvasive imaging in order to salvage viable myocardium. In contrast, in patients with a low to moderate risk of ACS, standard assessment includes symptoms and signs, physical examination, serial ECGs, and cardiac troponins, followed by optional functional testing (i.e., exercise ECG, rest and/or stress perfusion imaging, or stress echocardiography) [2,3]. However, this standard protocol may be associated with a lengthy stay in the ED or coronary care unit awaiting ECG or troponin changes or stress testing, leading to ED overcrowding and increased costs. Notably, cutting-edge CT (64-slice CT or newer generation CT scanners) has the capability to rapidly and reliably exclude non-ST-segment elevation ACS by directly visualizing the coronary arterial wall [2,3]. In addition, the recent approval of high-sensitivity troponins (hs-Tn) has overcome previous shortcomings of conventional troponin assays by allowing the detection of acute myocardial infarction (AMI) at an earlier stage. $[4,5]$. Thus, there may be uncertainty among ED physicians and cardiologists regarding the best option (i.e., anatomic (CT) versus hs-Tn with functional testing) for evaluating ED chest pain [6].

This review article summarizes insights from multiple studies regarding the strengths and shortcomings of coronary CTA over a traditional management protocol of ACP and provides an overview of basic $\mathrm{CT}$ techniques and characteristic $\mathrm{CT}$ findings of ACS. 


\section{Insights from Recent Randomized Controlled Trials Regarding Coronary CTA in the Evaluation of Acute Chest Pain}

\subsection{Randomized Controlled Trials Prior to the Era of High-Sensitivity Troponin}

The ROMICAT II and ACRIN trials reported very high negative predictive values for coronary CTA in excluding $>50 \%$ coronary stenosis in ED settings, demonstrating that this approach can lead to a more rapid and safer discharge from the ED $(47 \%$ vs. $12 \%$, $p<0.001$, and $49.6 \%$ vs. $22.7 \%, p<0.001$, respectively), a shorter the ED stay ( $8.6 \mathrm{~h}$ vs. $26.7 \mathrm{~h}$, $p<0.001$, and $18.0 \mathrm{~h}$ vs. $24.8 \mathrm{~h}, p<0.001$, respectively), and improved ED cost-effectiveness compared to the standard assessment $[7,8]$. However, there was no definite short-term outcome benefit in the $\mathrm{CT}$ arm compared to standard care in these trials. In contrast, the $\mathrm{CATCH}$ trial demonstrated a midterm outcome benefit (i.e., major adverse cardiac events, including cardiac death, AMI, unstable angina, and symptom-driven revascularization; $p=0.04$; HR: 0.36 (95\% CI: 0.16 to 0.95$)$ ) in the CT arm over the standard assessment [9]. The contradictory results between the CATCH (Denmark) and other trials may be caused by the time difference in defining outcome and different referral patterns compared to the US. The outcome was measured as a short-term parameter ( $<30$ days) in the ROMICAT II and ACRIN trials versus a midterm parameter in the CATCH trial (median, 18.7 months). The favorable results in $\mathrm{CT}$ outcome obtained from the $\mathrm{CATCH}$ trial may reflect the ability of CT to precisely identify obstructive and nonobstructive coronary artery disease. The identification of obstructive stenosis may have resulted in the better use of invasive coronary angiography and revascularization, leading to a decrease in episodes of unstable angina in the midterm to a greater extent than in the short term. Moreover, increased identification of nonobstructive coronary artery stenosis in the CT arm may have resulted in the greater use of statins [10]. However, there remains debate as to whether using a $\mathrm{CT}$ protocol causes increased downstream testing as coronary CTA has a nonnegligible false-positive rate, mainly due to blooming artifacts of calcified plaque. According to a meta-analysis of the outcomes of randomized controlled trials of coronary CTA in the ED, the use of coronary CTA was associated with decreased ED cost and length of stay but increased rates of coronary angiography and revascularization [11]. In summary, the use of a CT protocol in the ACP setting is associated with a reduced ED stay and/or costs compared to standard care with conventional troponins. However, further randomized trials are required to address whether there is an outcome benefit and any effects on downstream testing.

\subsection{Randomized Controlled Studies in the Era of High-Sensitivity Troponin}

The ROMICAT II, ACRIN, and CATCH trials were performed prior to the approval of hs-Tn. In contrast, the BEACON trial used hs-Tn and showed that there was no difference in the length of ED stay (both groups $=6.3 \mathrm{~h}$ ) between the $\mathrm{CT}$ and standard assessment groups [12]. These results may portend the decreased utility of coronary CTA in patients with a low risk of ACS and a normal hs-Tn level. In addition, the BEACON trial did not demonstrate a 30-day outcome benefit over standard care (CT and standard arm, 10\% vs. $9 \%$, respectively, $>0.05$ ). The negative result regarding a short-term outcome benefit is expected considering that a majority of the enrolled patients had a low pretest probability $(84 \%)$ in the trial [12]. However, it is unclear whether the identification of obstructive and nonobstructive coronary stenosis by CT might result in an outcome benefit during a longer follow-up in patients with an intermediate risk of ACS. It is hoped that the ongoing RAPID-CTCA trials will answer this question in the near future. In summary, the BEACON trial suggested that $\mathrm{CT}$ does not reduce ED stay length in the era of hs-Tn compared to standard care [13]. Thus, it is expected that hs-Tn may be useful to exclude ACS in a manner similar to the role of D-dimer in the evaluation of patients at low risk of pulmonary embolism in the ED. In addition, it remains unclear whether the use of a CT-driven protocol is beneficial in terms of outcome over standard assessment. 


\section{What Is the Proper Indication for Coronary CT in the Evaluation of Acute} Chest Pain?

\subsection{Definition of Pretest Probability for Acute Chest Pain}

Some clinical features are known to be valuable in differentiating ACP caused by ACS from other etiologies. For instance, pain aggravated by deep inspiration or pain reproducible on palpation are often caused by diseases other than ACS. Pain caused by ACS is often characterized by substernal heaviness or squeezing in nature, lasting more than $30 \mathrm{~min}$ and/or radiating to the neck or arms. However, precise discrimination of ACS from the other causes of ACP is often difficult based merely on the assessment of the patient's symptoms and signs [14]. There are multiple scoring systems used to predict the pretest probability of ACS in patients with ACP (e.g., TIMI, GRACE, HEART, EDACS score, and Diamond-Forrester criteria) [15-19]. Indeed, the previous randomized controlled trials used different tools in their studies. For instance, the ROMICAT II and BEACON trials used TIMI and TIMI and GRACE scores, respectively, while the CATCH trial used both the Diamond-Forrester criteria and TIMI risk score to assess pretest probability. However, the Diamond-Forrester criteria consist of patient age, sex, and the presence or absence of typical angina. The definition of typical angina should have all three characteristic components (substernal squeezing pain, aggravating and relieving factors). However, mitigating and/or aggravating factors are often absent in patients with ACS, rendering the scoring system less than optimal. Thus, application of the Diamond-Forrester criteria may be more suitable for patients with a concern for stable angina. Although each scoring system has different components, atypical or typical chest pain accompanied by a nondiagnostic ECG and normal initial troponins is commonly classified as a low or intermediate risk of ACS. Tatum et al. defined an intermediate risk of ACS as typical ACP lacking objective evidence of ACS (i.e., substernal ACP with squeezing or heaviness lasting $>30$ min but with no ischemic ECG abnormalities and troponin elevation). In addition, a low risk of ACS was defined as typical ACP lasting $<30$ min or atypical ACP lasting $>30$ min unaccompanied by ECG or troponin abnormalities [20]. The current consensus (Table 1) is that coronary CTA should be reserved for patients with an intermediate or low risk of ACS [21,22]. However, in the future, the role for coronary CTA may be more limited in the subgroup with normal hs-Tn and a low risk of ACS. Intermediate-risk patients with typical chest pain lasting $>30$ min but normal ECG and hs-Tn may be deemed suitable for coronary CTA in the future. Notably, there may be increasing subgroups with a mild or nonspecific increase in the level of hs-Tn that will be included in the indications for coronary CTA, a cohort in which conventional troponins level would presumably have been normal.

Table 1. Guidelines for the use of coronary CTA in patients with possible acute coronary syndrome (ACS) presenting without ST elevation.

\begin{tabular}{cl}
\hline & Indication \\
\hline \multirow{2}{*}{ 2020 ESC guidelines [21] } & $\begin{array}{l}\text { Low or intermediate risk of ACS with normal or } \\
\text { inconclusive troponin and ECG } \\
\text { Class I and level of evidence A }\end{array}$ \\
\hline \multirow{2}{*}{$2014 \mathrm{AHA} /$ ACC guidelines [22] } & $\begin{array}{l}\text { Normal ECG, normal cardiac troponin, and no history of } \\
\text { coronary artery disease } \\
\text { Class Ila and level of evidence A }\end{array}$ \\
\hline
\end{tabular}

\subsection{Contraindications to Coronary CTA}

Several patient-related conditions may preclude coronary CTA examination. These include hemodynamic instability, renal failure, known coronary artery disease, pregnancy, inability to control heart rate as can occur with arrhythmia, and a history of an allergic reaction to contrast materials. 


\section{Basic CT Techniques and Characteristic Coronary CT Findings of Acute Coronary Syndrome}

\subsection{Basics of Coronary CT Techniques}

To achieve sufficient image quality during coronary CTA, heart rate control should be optimized using beta-blockers. The target heart rate is different depending on the specific CT machine. In general, single-source and dual-source CT require a rate $<60$ and $<70 \mathrm{bpm} / \mathrm{min}$, respectively, to minimize coronary motion artifacts. In addition, sublingual nitroglycerin should be administered to dilate the coronary arteries, provided there is no contraindication such as hypotension or ongoing use of Sildenafil. In contrast to non-ECG gated chest $\mathrm{CT}$, coronary CTA is inevitably associated with a higher radiation exposure due to the low pitch mode used for ECG gating, especially with single-source CT. Thus, measures to reduce radiation exposure should be employed, especially in younger female patients. There are two basic options available to acquire coronary CTA, retrospective and prospective ECG gating. The former has greater radiation exposure as images are obtained throughout the cardiac cycle but has the advantage that it enables the acquisition of a cine loop, permitting assessment of left ventricular function [3]. Another important issue is whether a triple-rule-out examination is appropriate in patients with nonspecific ACP. Triple rule-out may be performed in order to simultaneously exclude aortic dissection, pulmonary embolism, and ACS in patients with nonspecific ACP. The field of view for dedicated coronary CTA typically includes the lower two-thirds of the chest. With this field of view, most aortic dissections and pulmonary emboli are demonstrated on dedicated coronary CTA [23]. Thus, a triple-rule-out should be avoided in younger female patients with nonspecific ACP due to the greater radiation exposure caused by wider z-axis coverage (Figure 1).

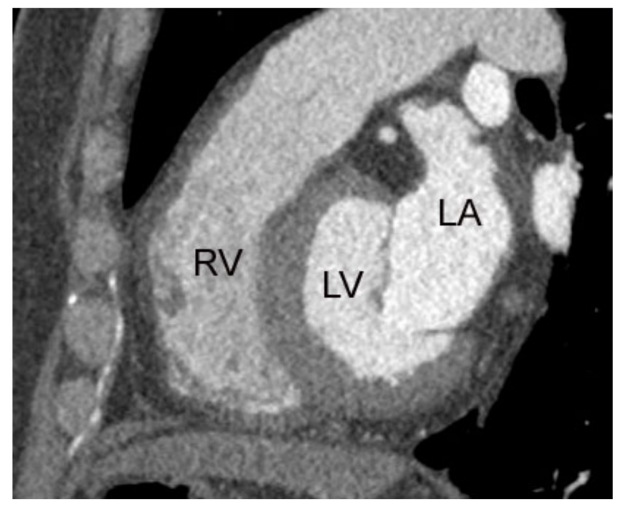

(a)

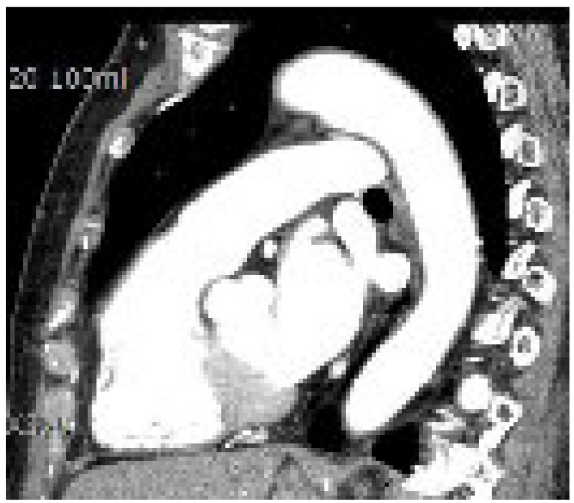

(b)

Figure 1. Typical z-axis coverage of dedicated coronary CTA versus triple-rule-out. (a) Z-axis coverage of dedicated coronary CTA includes the lower two-thirds of the entire chest. (b) The entire chest constitutes the z-axis coverage of the triple-rule-out CT. RV, LA, and LV indicate right ventricle, left atrium, and left ventricle, respectively.

\subsection{Coronary Artery Abnormalities of Acute Coronary Syndrome}

4.2.1. How to Identify Coronary Abnormalities on Coronary CTA Culprit Plaque of Type 1 Acute Myocardial Infarction

A Type 1 AMI is initiated by vulnerable plaque rupture resulting in obstructive coronary stenosis or occlusion. Thus, a culprit lesion is often characterized by a $\geq 70 \%$ stenosis with mixed or noncalcified plaque accompanied by positive remodeling, lowattenuation plaque, the napkin ring sign, and spotty calcification ( $<3 \mathrm{~mm}$ in diameter) on coronary CTA (Figure 2). Positive remodeling is defined as the adaptive outward growth of atherosclerotic plaque with a remodeling index $>1.1$ (the remodeling index = coronary artery diameter at the level of a plaque/coronary artery diameter at a nearby reference level without plaque). Low-attenuation plaque on CT is often defined by Hounsfield units 
$(\mathrm{HU})<30$, indicating a lipid core [24-26]. However, measurement of a low-attenuation plaque may be imprecise due to partial volume averaging depending on the amount of intraluminal coronary enhancement on $\mathrm{CT}$, rendering this finding less specific. The napkin ring sign is defined by a CT plaque demonstrating inner lower and outer higher attenuation (i.e., inhomogeneous plaque with lower internal attenuation) [27]. A thin-cap fibroatheroma is a major feature of vulnerable plaque on microscopic examination. Because the thickness of the thin-cap fibroatheroma is $<65 \mu \mathrm{m}$, it cannot be directly visualized on CT due to limits of spatial resolution [28]. A thin-cap fibroatheroma can be presumed to be present on CT based on the identification of the napkin ring sign. However, even the combination of obstructive stenosis and vulnerable CT features does not necessarily indicate a culprit lesion of ACS.

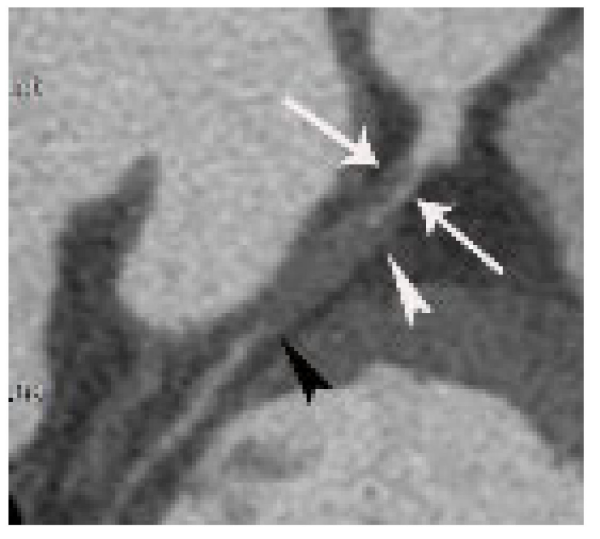

(a)

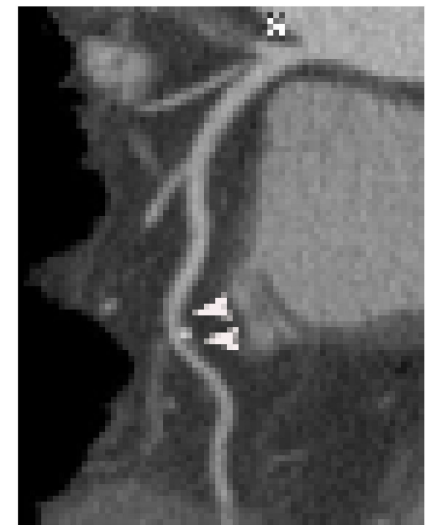

(b)

Figure 2. Difference between culprit (a) and nonculprit plaque (b) on coronary CTA. (a) Note the total occlusion (arrowheads) by noncalcified plaque with vulnerable CT features (positive remodeling and napkin ring sign appearance (arrows)) in the proximal left circumflex artery in a patient with AMI, showing it to be the culprit lesion. (b) In contrast, $<50 \%$ stenosis with mixed plaque without vulnerable CT features (arrowheads) is noted in the mid left anterior descending coronary artery in a nonculprit lesion.

This raises the issue of how to discriminate a culprit lesion from an innocent bystander lesion with vulnerable CT features. During an episode of ACS, two main changes occur within a precursor lesion with vulnerable features [26]. The first alteration is the tearing of the thin cap with the expulsion of the lipid core of plaque into the bloodstream. The second change is sudden thrombus formation within the ruptured coronary segment. Chun et al. [26] reported that there was no difference in the prevalence of the CT signs of low-attenuation plaque and the napkin ring sign among precursor and culprit lesions in patients with ACS. Thus, they assumed that the first changes may be subtle and likely below the spatial resolution of CT. In contrast, total occlusion suggesting thrombus formation, and myocardial hypoattenuation suggesting myocardial necrosis is more common in a culprit lesion compared to a precursor lesion on CT [26]. The coronary occlusion is due to thrombosis after the episode of ACS. Several points (Figure 3) are helpful to differentiate a culprit lesion from a nonculprit obstructive coronary stenosis on CT, including (1) vulnerable plaque features, (2) myocardial hypoattenuation or wall motion abnormality, and (3) epicardial fat stranding. 


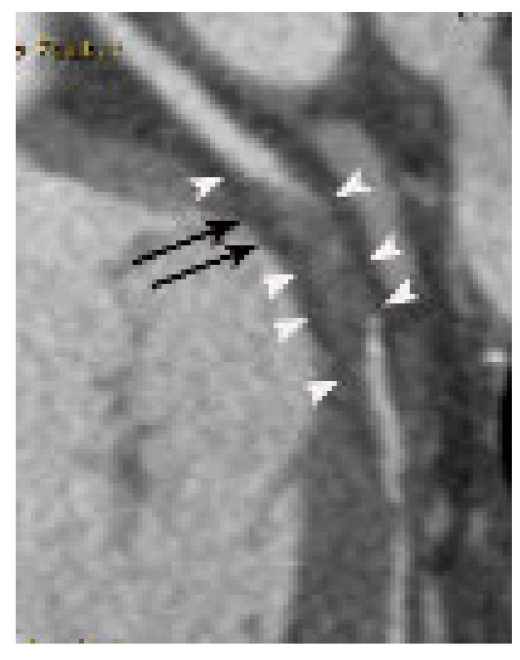

(a)

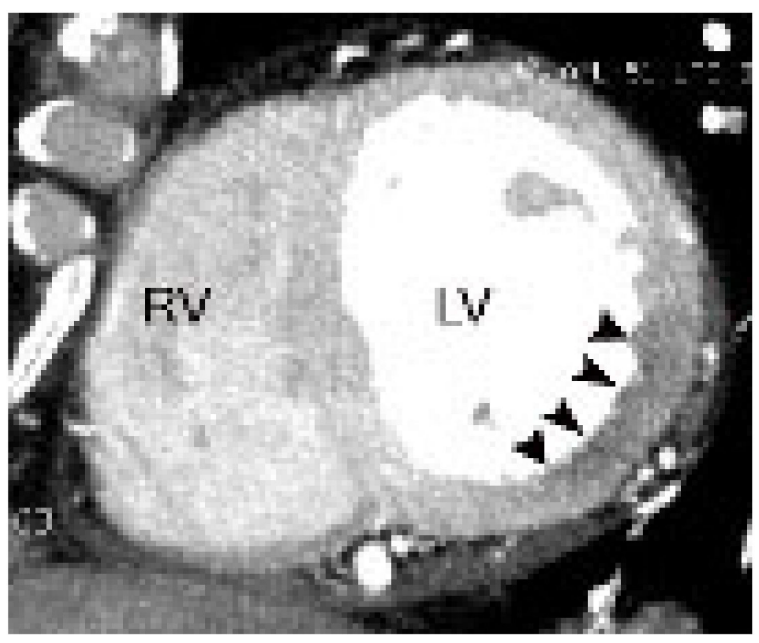

(b)

Figure 3. Fat stranding in a 75-year-old male patient with acute myocardial infarction referable to the left circumflex coronary artery. (a) Total occlusion (arrowheads) is noted in the proximal portion of the distal left circumflex coronary artery. Note the positive remodeling and subtle irregular lines (arrows) at the epicardial fat interface near the occluded coronary wall, suggesting epicardial fat stranding. (b) Subendocardial hypoattenuation (arrowheads) is demonstrated in the territory of the left ventricle on a short-axis view, consistent with myocardial necrosis. RV and LV indicate right and left ventricle, respectively.

Culprit Plaque of Type 2 Acute Myocardial Infarction

Type 2 AMI is caused by a mismatch of blood supply and demand. Type 2 AMI is often referred to as myocardial infarction with nonobstructive coronary arteries (MINOCA) (Figure 4) [29]. MINOCA may be due to prolonged coronary spasm, spontaneous thrombolysis, and coronary embolism. Based on the theory of spontaneous thrombolysis in the MINOCA, an initial event assumed to be similar to that of typical vulnerable plaque rupture results in obstructive coronary stenosis. However, exaggerated spontaneous thrombolysis may be endogenously initiated in patients with MINOCA, leading to evolution to nonobstructive coronary stenosis. The coronary spasm in MINOCA may extend for a sufficient length of time to result in myocardium necrosis. Coronary angiography or CT may be normal or demonstrate $<50 \%$ stenosis after the spasm is relieved [29].

Culprit Plaque in Unstable Angina

ACS consists of ST-elevated AMI, non-ST-elevated AMI, and unstable angina. The CT features of unstable angina (Figure 5) are similar to those of type 1 AMI but lack the wall motion abnormality and myocardial hypoattenuation in the affected arterial territory as there is no myocardial necrosis. 


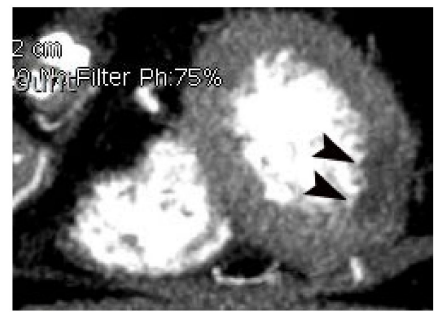

(a)

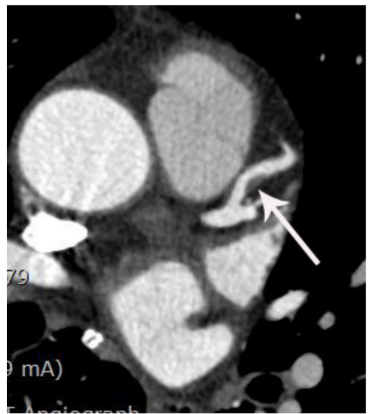

(d)

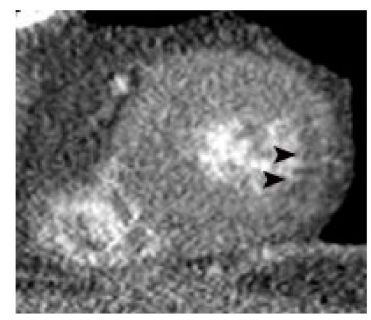

(b)

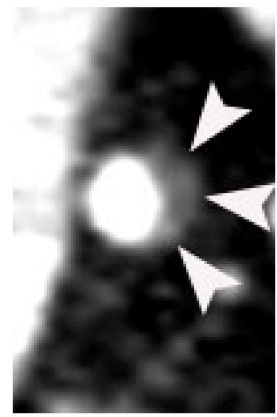

(e)

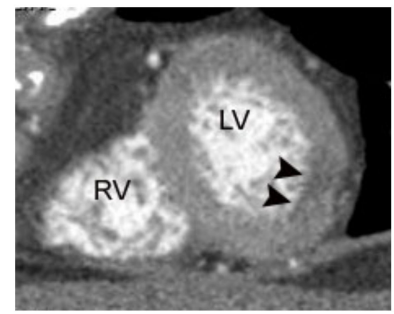

(c)

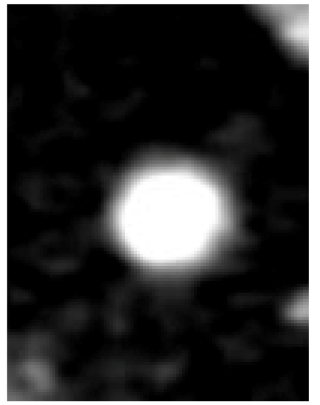

(f)

Figure 4. A case of myocardial infarction with nonobstructive coronary arteries (MINOCA) in a 66-year-old female patient. The patient presented to the emergency department (ED) with acute chest pain. Focal hypoattenuation (arrowheads in (a)) in the lateral wall of the apex of the left ventricle is noted on a CT short-axis view. There is akinesia in the same location on a short-axis systolic image (b). Note the absence of wall thickening in this area on the systolic image compared to the diastolic image $(\mathbf{b}, \mathbf{c})$. However, the coronary arteries were normal other than nonobstructive coronary stenosis (arrow in (d)) with noncalcified plaque and positive remodeling in the proximal left anterior descending coronary artery on an axial coronary CT image (d). Note the noncalcified plaque with positive remodeling on an en face view (arrowheads in (e)) compared to the normal reference level (f). Subsequent coronary angiography confirms the CT findings (not shown). RV and LV indicate right and left ventricle, respectively.

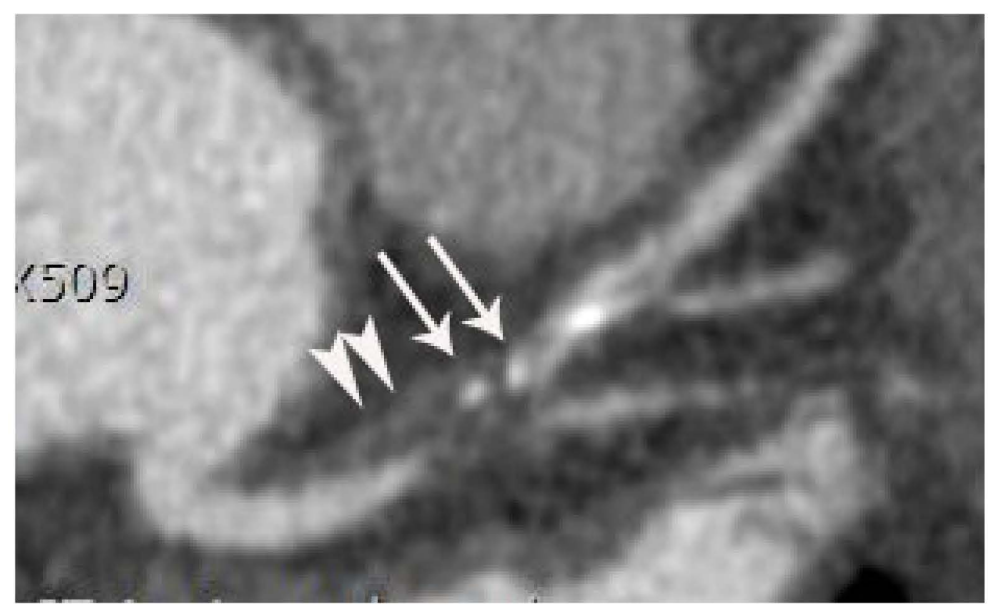

(a)

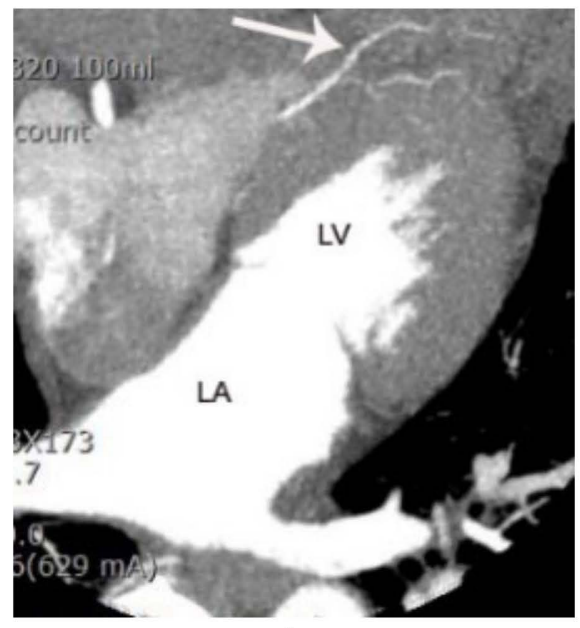

(b)

Figure 5. A case of unstable angina in a 61-year-old male patient with crescendo angina. (a) Curved multiplanar reformatted image shows near-total occlusion in the distal left main coronary artery. Note the napkin ring sign (arrowheads), positive remodeling, and spotty calcifications (arrows). However, there is no myocardial hypoattenuation in the left ventricle on a four-chamber image. Note a collateral artery (arrow in (b)) from the posterior descending coronary artery coursing to the distal left anterior descending coronary artery. LV and LA indicate left ventricle and atrium, respectively. 


\subsection{Epicardial Fat Abnormality of Acute Coronary Syndrome}

Plaque inflammation is an important factor underlying ACS (i.e., vulnerable plaque rupture) resulting in inflammatory thinning of the fibrous cap. Previous studies have indicated that there is inflammatory cell infiltration in the media, adventitia, and epicardial fat as well as intima in patients with ACS [30-32]. Thus, epicardial inflammation may also be demonstrated on coronary $\mathrm{CT}$ as epicardial fat stranding (Figure 3). In patients without ACS, a sharp demarcation between the coronary artery wall and epicardial fat is present, even in plaques with positive remodeling. In contrast, in some patients with ACS $(<50 \%)$, this sharp demarcation may be lost resulting in irregularity and obscuration of epicardial fat [33].

\subsection{Myocardial Abnormalities of Acute Coronary Syndrome}

Evaluation for wall motion abnormalities and hypoattenuation in the left ventricle should be performed in all patients with suspicion for ACS. Notably, identification of wall motion abnormality or hypoattenuation along a specific coronary artery territory is valuable in cases with nondiagnostic coronary artery findings on CT due to coronary motion or blooming artifact [34]. It is important to understand the typical appearance of an arterial territory on a short-axis view (Figures 6 and 7) as its evaluation can enhance diagnostic confidence for AMI if there is an obstructive coronary stenosis with vulnerable plaque features in a matching coronary artery territory. Subendocardial hypoattenuation may be present in many cases with AMI on CT. The HU measurement within the area demonstrating hypoattenuation is often less than half that of an area with normal attenuation $[35,36]$. Transmural hypoattenuation is quite rare as coronary CTA is not performed in most patients with ST-elevated AMI. However, subendocardial hypoattenuation is often demonstrated in patients with non-ST-elevated AMI, suggesting myocardial necrosis and portending a worse prognosis. Hypoattenuation caused by AMI should be differentiated from artifact and chronic myocardial infarction (CMI) on CT. Cases with CMI (Figure

8) have fat attenuation on precontrast $\mathrm{CT}$ and are accompanied by myocardial thinning, while there is an absence of thinning in cases with AMI (Figures 6 and 7). Hypoattenuation secondary to motion or beam hardening artifact typically does not conform to a specific vascular territory.

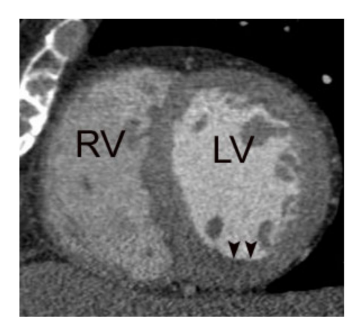

(a)

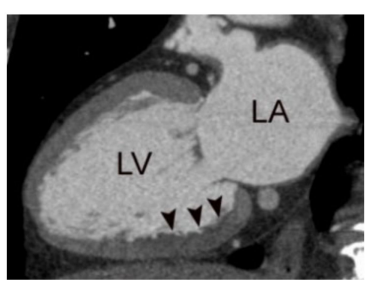

(d)

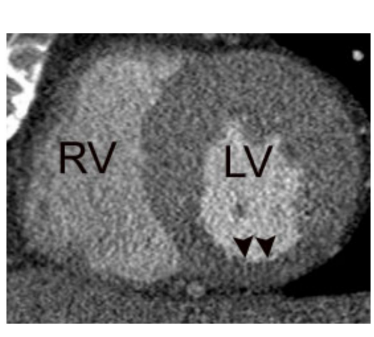

(b)

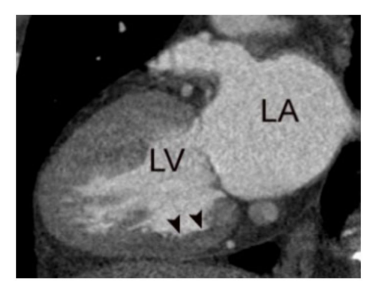

(e)

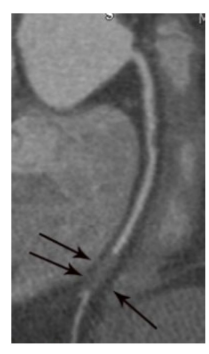

(c)

Figure 6. A case of acute myocardial infarction in a 60-year-old male patient. A. Note the akinesia (arrowheads) in the inferior wall of the mid to basal left ventricle in diastolic (a,d) and systolic images (b,e). Note the total occlusion in the distal right coronary artery (arrows in (c)) with positive remodeling and fat stranding, resulting in an irregular interface between the coronary wall and epicardial fat. RV, LA, and LV indicate right ventricle, left atrium, and left ventricle, respectively. 


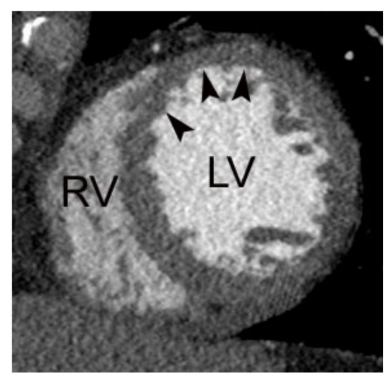

(a)

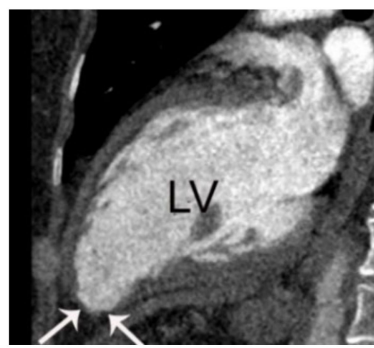

(d)

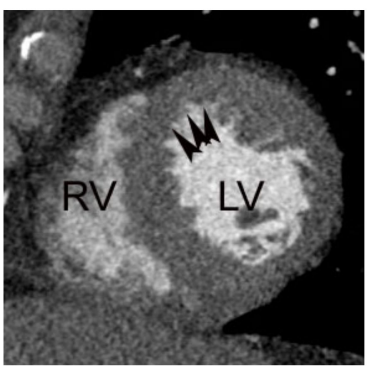

(b)

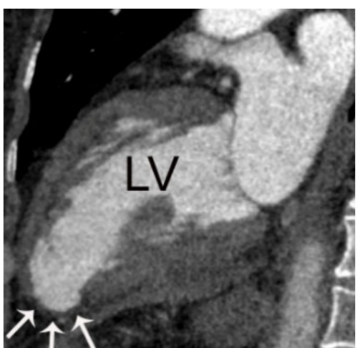

(e)

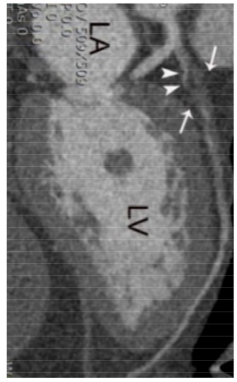

(c)

Figure 7. A case of acute myocardial infarction in a 61-year-old male patient. (a) Note the akinesia in the left anterior descending coronary artery territory in diastolic $(\mathbf{a}, \mathbf{d})$ and systolic images $(\mathbf{b}, \mathbf{e})$ (arrowheads or arrows). Note also the total occlusion of the proximal left anterior descending coronary artery by noncalcified plaque (arrows in (c)) with positive remodeling, low-attenuation plaque, and a napkin ring sign (arrowheads in (c)). RV, LA, and LV indicate right ventricle, left atrium, and left ventricle, respectively.

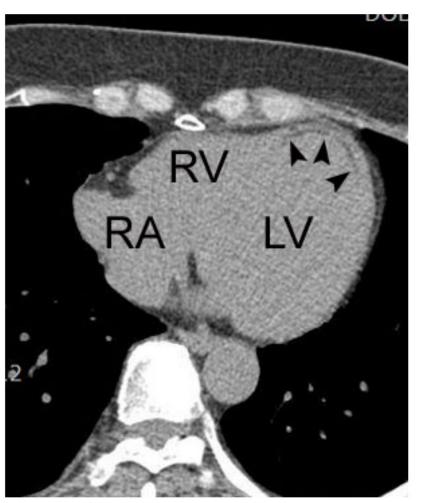

(a)

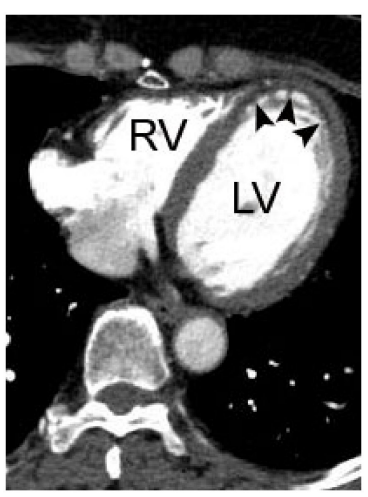

(b)

Figure 8. A case of chronic myocardial infarction in the left anterior descending coronary artery in a 63-year-old female patient. Note the subendocardial fat and myocardial thinning in the left ventricular apex (arrowheads in (a,b)). RV, RA, and LV indicate right ventricle, right atrium, and left ventricle, respectively.

\subsection{Complications of Acute Myocardial Infarction}

Complications of AMI include pulmonary edema, acute mitral regurgitation, ventricular septal defect, and free-wall rupture [36]. Acute mitral regurgitation can occur if AMI affecting the left circumflex coronary artery results in papillary muscle necrosis [37]. The posteromedial papillary muscle is often involved in such cases due to its solitary blood supply. Free-wall rupture caused by AMI is a rare life-threatening complication presenting with shock (Figure 9) [38]. 


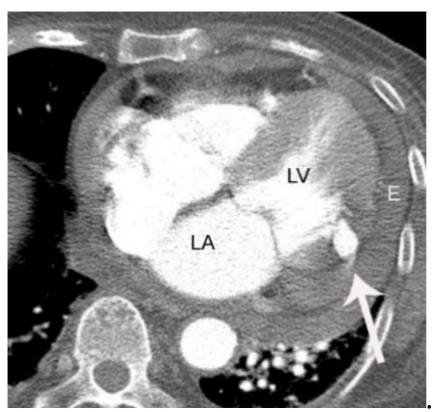

Figure 9. A case of left ventricular free-wall rupture in an 84-year-old male patient who presented with cardiogenic shock. Note the rupture site (arrow) and pericardial hematoma (E). LA and LV indicate left atrium and ventricle, respectively.

\subsection{Which Vulnerable Plaque Will Eventually Lead to Acute Coronary Syndrome?}

Not all vulnerable plaques eventually rupture and cause ACS. However, one study indicated that the presence of plaque progression defined as an increasing degree of coronary stenosis or remodeling index, or increasing size of the lipid core on follow-up coronary CT was associated with an increased risk of a future cardiac event [39]. Thus, the progression of vulnerable plaque morphology may be more important in predicting a future episode of ACS than vulnerable plaque morphology visualized on CT at a single point in time (Figure 10).
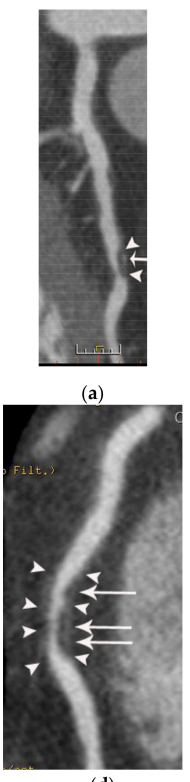

(d)

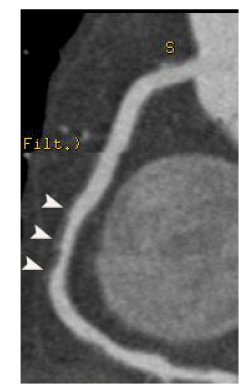

(b)

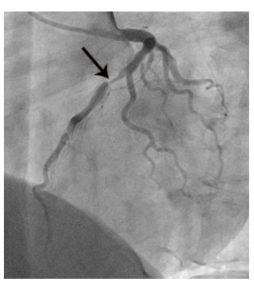

(e)
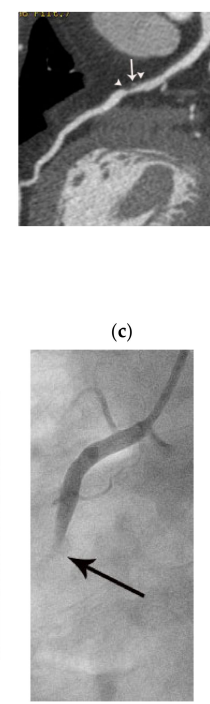

(f)

Figure 10. A 46-year-old man presenting with ACS. Coronary CTA was obtained due to multiple cardiac risk factors. Approximately 70\% stenoses with mixed plaque (a) and 40-50\% stenosis with noncalcified plaque (b) are noted in the mid left anterior descending and mid right coronary artery, respectively. At the 12-month follow-up coronary CTA, the stenosis in the mid left anterior descending coronary artery shows no definite change (c). In contrast, note the progression of plaque (d) in the mid right coronary artery in the degree of coronary stenosis (about a 70\% stenosis), the increase in the positive remodeling index (arrowheads in (d)) and the low-attenuation plaque/napkin ring sign (arrows in (d)). Invasive coronary angiography was recommended in this patient, but the patient refused and was lost to follow-up. He presented to the ED with acute chest pain 2 months later. Emergent coronary angiography showed total occlusion indicating a culprit lesion in the same location as the vulnerable plaque in the mid right coronary artery (arrow in (f)). In contrast, the stenosis in the proximal middle left anterior descending coronary artery (nonculprit lesion) showed no interval change (arrow in (e)). 


\subsection{Alternative Diagnosis}

Clinically important alternative diagnoses (e.g., aortic dissection, pulmonary embolism, or stress-induced cardiomyopathy) can be made on coronary CTA [2]. This is a compelling advantage of anatomical imaging over functional testing (Figures 11-14).

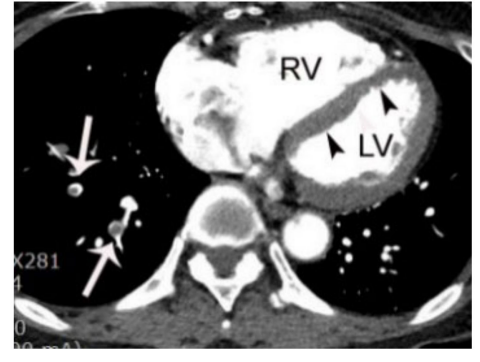

(a)

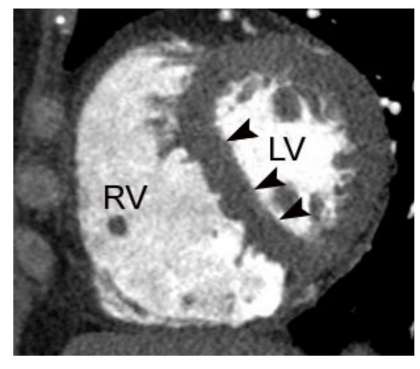

(b)

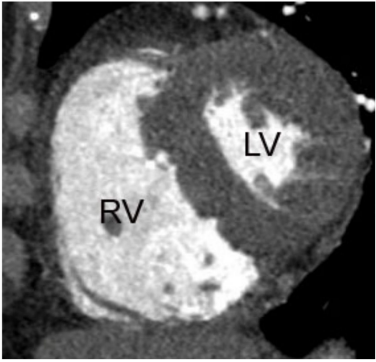

(c)

Figure 11. A case of submassive pulmonary embolism in a 66-year-old female patient shown on coronary CTA. (a) Multiple pulmonary emboli (arrows) are noted in the right lower lobe on an axial CT image using a wide field of view. Note marked dilatation of the right ventricle and straightening of the interventricular septum (arrowheads) suggesting increased right ventricular pressure. A decrease in the contractile function of the right ventricle can be assessed in this patient due to the use of retrospective ECG gating. Note the lack of change in the right ventricular volume between the diastolic (b) and systolic (c) short-axis images indicating right ventricular dysfunction. RV and LV indicate right and left ventricle, respectively.

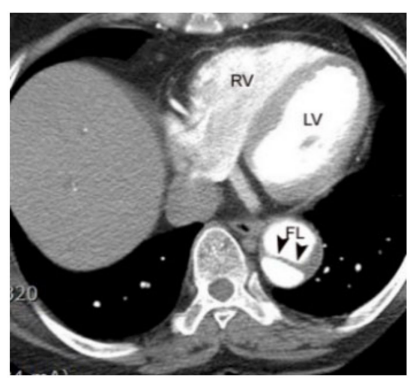

(a)

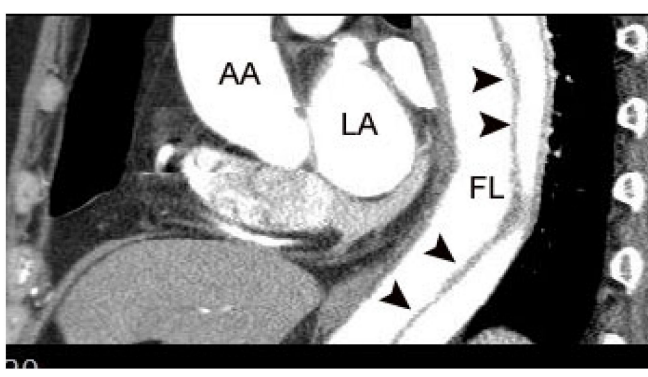

(b)

Figure 12. A case of type $B$ aortic dissection identified on coronary CTA. Note the intimal flap within the descending thoracic aorta (arrowheads in $(\mathbf{a}, \mathbf{b})$ ) RV, LA, LV, AA, FL indicate right ventricle, left atrium, left ventricle, ascending aorta, and false lumen, respectively.

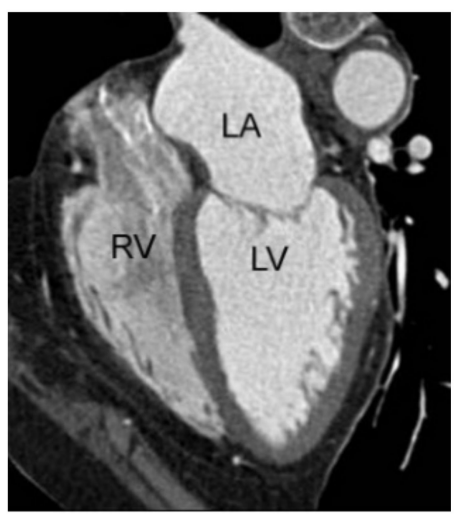

(a)

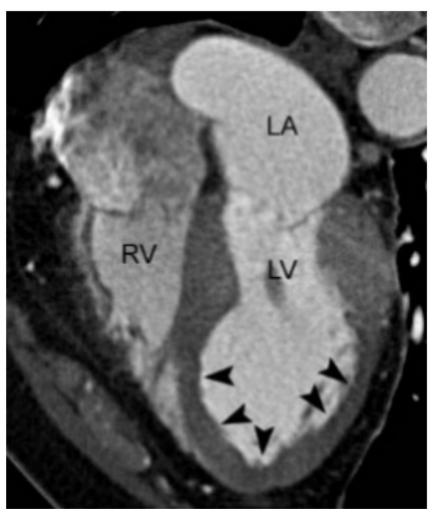

(b)

Figure 13. A case of stress-induced cardiomyopathy in a 65-year-old female patient identified on coronary CTA. Typical apical ballooning (arrowheads in (b)) is noted on a systolic four-chamber image in comparison to a diastolic image (a). There was no obstructive coronary artery stenosis on coronary CTA or invasive coronary angiography (not shown). 


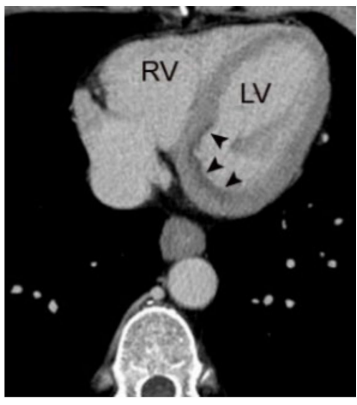

(a)

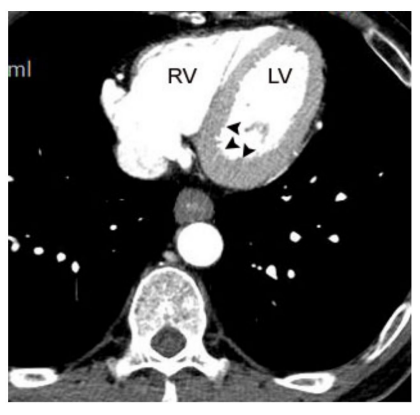

(b)

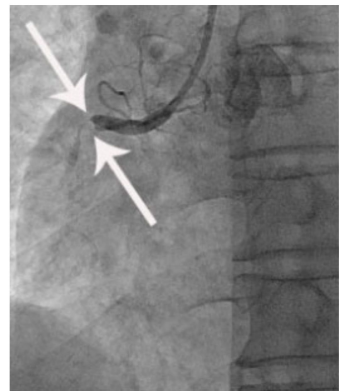

(c)

Figure 14. A case of acute myocardial infarction in a 60-year-old male patient with hepatocellular carcinoma identified on non-ECG gated CT. (a) Abdomen CT was performed in this patient for routine follow-up of hepatocellular carcinoma. Note the typical subendocardial hypoattenuation without myocardial thinning (arrowheads) in the right coronary artery territory (basal left ventricle). HU of the low and normal attenuation areas in the left ventricle were 45 and 109, respectively. The constellation of CT findings was consistent with AMI in the right coronary artery territory, although there was no corroborating information regarding clinical history, ECG, and troponin level. (b) Note the normal attenuation in the same area (arrowheads) on an axial CT image 2 months previously. The ED physician was notified of the CT findings and subsequently confirmed ischemic ECG changes and an elevated troponin level. (c) Emergent coronary angiography confirmed total occlusion of the proximal right coronary artery (arrows), and subsequent revascularization was performed.

\subsection{Incidental Identification of Acute Myocardial Infarction on Nongated Abdomen or Chest CT}

Although nongated chest or abdomen CT is not indicated in the evaluation of ACS, an occasional AMI is incidentally identified on nongated CT (Figure 14). Thus, caution should be exercised to avoid overlooking this important finding, and the myocardium should be inspected as part of all such studies [36,40].

\subsection{Ability of Coronary CTA to Predict Future Cardiac Event}

A previous study indicated that the type (i.e., mixed or noncalcified plaque rather than calcified plaque) and extent of coronary plaque as well as the presence of wall motion abnormalities on the coronary CTA are associated with increased risk of a future cardiac event in the patients presenting with ACP [41]. The potential for coronary CTA to predict future cardiac events may be improved if a better correlation of high-risk plaque can be established with findings on optical coherence tomography [42].

\section{Future Directions}

The lack of functional information is an important shortcoming of coronary CTA. In fact, the application of coronary CTA in the ED may have the potential to increase the use of invasive coronary angiography due to its non-negligible false-positive diagnosis rate. However, recent studies suggest that the shortcoming can be overcome by adding a CT fractional flow reserve to coronary CTA, which has shown favorable results in ED settings as well as in planning for complex percutaneous coronary intervention $[43,44]$. In addition, ongoing advances in CT hardware and software, including the application of dual-energy CT and artificial intelligence approaches, will likely increase the diagnostic accuracy of CT in ED settings.

\section{Conclusions}

Coronary CTA is an attractive option to exclude ACS in patients with a low to intermediate risk due to its high negative predictive value. A precise understanding of the typical CT findings of ACS can assist interpreting physicians in providing a rapid and precise ACS diagnosis leading to improvement in patient outcome. 
Author Contributions: Conceptualization, M.J.S., and S.M.Y.; methodology, M.J.S. and S.M.Y.; software, M.J.S.; validation, M.J.S., and S.M.Y.; formal analysis, M.J.S., and S.M.Y.; investigation, D.L., M.J.S. and S.M.Y.; resources, M.J.S. and S.M.Y.; data curation, M.J.S., and S.M.Y.; writing-original draft preparation, D.L.; writing-review and editing, M.J.S., D.L., S.M.Y., H.Y.L., E.J.C., I.S.S., and C.S.W.; visualization, S.M.Y.; supervision, S.M.Y.; project administration, S.M.Y. All authors have read and agreed to the published version of the manuscript.

Funding: This research received no external funding.

Conflicts of Interest: The authors declare no conflict of interest.

Dataset License: License under which the dataset is made available (CC0, CC-BY, CC-BY-SA, CC-BYNC, etc.)

\section{References}

1. Bhuiya, F.A.; Pitts, S.R.; McCaig, L.F. Emergency department visits for chest pain and abdominal pain: United States, 1999-2008. NCHS Data Brief 2010, 43, 1-8.

2. Lee, H.Y.; Yoo, S.M.; White, C.S. Coronary CT angiography in emergency department patients with acute chest pain: Triple rule-out protocol versus dedicated coronary CT angiography. Int. J. Cardiovasc. Imaging 2009, 25, 319-326. [CrossRef]

3. Yoo, S.M.; Rho, J.Y.; Lee, H.Y.; Song, I.S.; Moon, J.Y.; White, C.S. Current Concepts in Cardiac CT Angiography for Patients With Acute Chest Pain. Korean Circ. J. 2010, 40, 543-549. [CrossRef]

4. Reichlin, T.; Hochholzer, W.; Bassetti, S.; Steuer, S.; Stelzig, C.; Hartwiger, S.; Biedert, S.; Schaub, N.; Buerge, C.; Potocki, M.; et al. Early diagnosis of myocardial infarction with sensitive cardiac troponin assays. N. Engl. J. Med. 2009, 361, 858-867. [CrossRef] [PubMed]

5. Keller, T.; Zeller, T.; Peetz, D.; Tzikas, S.; Roth, A.; Czyz, E.; Bickel, C.; Baldus, S.; Warnholtz, A.; Fröhlich, M.; et al. Sensitive troponin I assay in early diagnosis of acute myocardial infarction. N. Engl. J. Med. 2009, 361, 868-877. [CrossRef] [PubMed]

6. Yang, S.; Manjunath, L.; Willemink, M.J.; Nieman, K. The role of coronary CT angiography for acute chest pain in the era of high-sensitivity troponins. J. Cardiovasc. Comput. Tomogr. 2019, 13, 267-273. [CrossRef] [PubMed]

7. Litt, H.; Gatsonis, C.; Snyder, B.; Singh, H.; Miller, C.D.; Entrikin, D.W.; Leaming, J.M.; Gavin, L.J.; Pacella, C.B.; Hollander, J.E. CT angiography for safe discharge of patients with possible acute coronary syndromes. N. Engl. J. Med. 2012, 366, 1393-1403. [CrossRef]

8. Hoffmann, U.; Truong, Q.A.; Schoenfeld, D.A.; Chou, E.T.; Woodard, P.K.; Nagurney, J.T.; Pope, J.H.; Hauser, T.H.; White, C.S.; Weiner, S.G.; et al. Coronary CT angiography versus standard evaluation in acute chest pain. N. Engl. J. Med. 2012, 367, 299-308. [CrossRef]

9. $\quad$ Linde, J.J.; Hove, J.D.; Sørgaard, M.; Kelbæk, H.; Jensen, G.B.; Kühl, J.T.; Hindsø, L.; Køber, L.; Nielsen, W.B.; Kofoed, K.F. Long-Term Clinical Impact of Coronary CT Angiography in Patients with Recent Acute-Onset Chest Pain: The Randomized Controlled CATCH Trial. JACC Cardiovasc. Imaging 2015, 8, 1404-1413. [CrossRef]

10. Newby, D.E.; Gray, A.J. CATCH a Glimpse of the Future. JACC Cardiovasc. Imaging 2015, 8, 1414-1416. [CrossRef]

11. Hulten, E.; Pickett, C.; Bittencourt, M.S.; Villines, T.C.; Petrillo, S.; Di Carli, M.F.; Blankstein, R. Outcomes after coronary computed tomography angiography in the emergency department: A systematic review and meta-analysis of randomized, controlled trials. J. Am. Coll. Cardiol. 2013, 61, 880-892. [CrossRef]

12. Dedic, A.; Lubbers, M.M.; Schaap, J.; Lammers, J.; Lamfers, E.J.; Rensing, B.J.; Braam, R.L.; Nathoe, H.M.; Post, J.C.; Nielen, T.; et al. Coronary CT Angiography for Suspected ACS in the Era of High-Sensitivity Troponins: Randomized Multicenter Study. J. Am. Coll. Cardiol. 2016, 67, 16-26. [CrossRef] [PubMed]

13. Gray, A.J.; Roobottom, C.; Smith, J.E.; Goodacre, S.; Oatey, K.; O’Brien, R.; Storey, R.F.; Na, L.; Lewis, S.C.; Thokala, P.; et al. The RAPID-CTCA trial (Rapid Assessment of Potential Ischaemic Heart Disease with CTCA)-A multicentre parallelgroup randomised trial to compare early computerised tomography coronary angiography versus standard care in patients presenting with suspected or confirmed acute coronary syndrome: Study protocol for a randomised controlled trial. Trials 2016, 17, 579. [PubMed]

14. Alderwish, E.; Schultz, E.; Kassam, Z.; Poon, M.; Coplan, N. Evaluation of acute chest pain: Evolving paradigm of coronary risk scores and imaging. Rev. Cardiovasc. Med. 2019, 20, 231-244. [PubMed]

15. Antman, E.M.; Cohen, M.; Bernink, P.J.; McCabe, C.H.; Horacek, T.; Papuchis, G.; Mautner, B.; Corbalan, R.; Radley, D.; Braunwald, E. The TIMI risk score for unstable angina/non-ST elevation MI: A method for prognostication and therapeutic decision making. JAMA 2000, 284, 835-842. [CrossRef]

16. Granger, C.B.; Goldberg, R.J.; Dabbous, O.; Pieper, K.S.; Eagle, K.A.; Cannon, C.P.; Van De Werf, F.; Avezum, Á.; Goodman, S.G.; Flather, M.D.; et al. Predictors of hospital mortality in the global registry of acute coronary events. Arch. Intern Med. 2003, 163, 2345-2353. [CrossRef]

17. Six, A.J.; Backus, B.E.; Kelder, J.C. Chest pain in the emergency room: Value of the HEART score. Neth. Heart J. 2008, 16, 191-196. [CrossRef] [PubMed] 
18. Than, M.P.; Flaws, D.; Sanders, S.; Doust, J.; Glasziou, P.; Kline, J.; Aldous, S.; Troughton, R.; Reid, C.; Parsonage, W.A.; et al. Development and validation of the Emergency Department Assessment of Chest pain Score and $2 \mathrm{~h}$ accelerated diagnostic protocol. Emerg. Med. Australas. 2014, 26, 34-44. [CrossRef]

19. Diamond, G.A.; Forrester, J.S. Analysis of probability as an aid in the clinical diagnosis of coronary-artery disease. N. Engl. J. Med. 1979, 300, 1350-1358. [CrossRef]

20. Tatum, J.L.; Jesse, R.L.; Kontos, M.C.; Nicholson, C.S.; Schmidt, K.L.; Roberts, C.S.; Ornato, J.P. Comprehensive strategy for the evaluation and triage of the chest pain patient. Ann. Emerg. Med. 1997, 29, 116-125. [CrossRef]

21. Collet, J.P.; Thiele, H.; Barbato, E.; Barthélémy, O.; Bauersachs, J.; Bhatt, D.L.; Dendale, P.; Dorobantu, M.; Edvardsen, T.; Folliguet, T.; et al. 2020 ESC Guidelines for the management of acute coronary syndromes in patients presenting without persistent ST-segment elevation. Eur. Heart J. 2020. [CrossRef]

22. Amsterdam, E.A.; Wenger, N.K.; Brindis, R.G.; Casey, D.E., Jr.; Ganiats, T.G.; Holmes, D.R., Jr.; Jaffe, A.S.; Jneid, H.; Kelly, R.F.; Kontos, M.C.; et al. 2014 AHA/ACC guideline for the management of patients with non-ST-elevation acute coronary syndromes: A report of the American College of Cardiology/American Heart Association Task Force on Practice Guidelines. Circulation 2014, 130, e344-e426. [PubMed]

23. Lee, H.Y.; Song, I.S.; Yoo, S.M.; Rho, J.Y.; Moon, J.Y.; Kim, I.J.; Lim, S.W.; Sung, J.H.; Cha, D.H.; White, C.S. Rarity of isolated pulmonary embolism and acute aortic syndrome occurring outside of the field of view of dedicated coronary CT angiography. Acta Radiol. 2011, 52, 378-384. [CrossRef] [PubMed]

24. Yoo, S.M.; Chun, E.J.; Lee, H.Y.; Min, D.; White, C.S. Computed Tomography Diagnosis of Nonspecific Acute Chest Pain in the Emergency Department: From Typical Acute Coronary Syndrome to Various Unusual Mimics. J. Thorac. Imaging 2017, 32, 26-35. [CrossRef]

25. Motoyama, S.; Kondo, T.; Sarai, M.; Sugiura, A.; Harigaya, H.; Sato, T.; Inoue, K.; Okumura, M.; Ishii, J.; Anno, H.; et al. Multislice computed tomographic characteristics of coronary lesions in acute coronary syndromes. J. Am. Coll. Cardiol. 2007, 50, 319-326. [CrossRef]

26. Chun, E.J.; Han, J.H.; Yoo, S.M.; Lee, H.Y.; Song, I.S.; White, C.S. Differences in the CT findings between vulnerable plaque and culprit lesions in acute coronary syndrome. J. Cardiovasc. Comput. Tomogr. 2018, 12, 115-117. [CrossRef]

27. Maurovich-Horvat, P.; Hoffmann, U.; Vorpahl, M.; Nakano, M.; Virmani, R.; Alkadhi, H. The napkin-ring sign: CT signature of high-risk coronary plaques? JACC Cardiovasc. Imaging 2010, 3, 440-444. [CrossRef] [PubMed]

28. Ito, T.; Terashima, M.; Kaneda, H.; Nasu, K.; Matsuo, H.; Ehara, M.; Kinoshita, Y.; Kimura, M.; Tanaka, N.; Habara, M.; et al. Comparison of in vivo assessment of vulnerable plaque by 64-slice multislice computed tomography versus optical coherence tomography. Am. J. Cardiol. 2011, 107, 1270-1277. [CrossRef]

29. Yoo, S.M.; Jang, S.; Kim, J.A.; Chun, E.J. Troponin-Positive Non-Obstructive Coronary Arteries and Myocardial Infarction with Non-Obstructive Coronary Arteries: Definition, Etiologies, and Role of CT and MR Imaging. Korean J. Radiol. 2020, 21, 1305-1316. [CrossRef] [PubMed]

30. Laine, P.; Kaartinen, M.; Penttilä, A.; Panula, P.; Paavonen, T.; Kovanen, P.T. Association between myocardial infarction and the mast cells in the adventitia of the infarct-related coronary artery. Circulation 1999, 99, 361-369. [CrossRef]

31. Moreno, P.R.; Purushothaman, K.R.; Fuster, V.; O'Connor, W.N. Intimomedial interface damage and adventitial inflammation is increased beneath disrupted atherosclerosis in the aorta: Implications for plaque vulnerability. Circulation 2002, 105, $2504-2511$. [CrossRef] [PubMed]

32. Antonopoulos, A.S.; Sanna, F.; Sabharwal, N.; Thomas, S.; Oikonomou, E.K.; Herdman, L.; Margaritis, M.; Shirodaria, C.; Kampoli, A.-M.; Akoumianakis, I.; et al. Detecting human coronary inflammation by imaging perivascular fat. Sci. Transl. Med. 2017, 9, eaal2658. [CrossRef] [PubMed]

33. Hedgire, S.; Baliyan, V.; Zucker, E.J.; Bittner, D.O.; Staziaki, P.V.; Ap Takx, R.; Scholtz, J.-E.; Meyersohn, N.; Hoffmann, U.; Ghoshhajra, B. Perivascular Epicardial Fat Stranding at Coronary CT Angiography: A Marker of Acute Plaque Rupture and Spontaneous Coronary Artery Dissection. Radiology 2018, 287, 808-815. [CrossRef] [PubMed]

34. Seneviratne, S.K.; Truong, Q.A.; Bamber, F.; Rogers, I.S.; Shapiro, M.D.; Schlett, C.L.; Chae, C.U.; Cury, R.; Abbara, S.; Brady, T.J.; et al. Incremental diagnostic value of regional left ventricular function over coronary assessment by cardiac computed tomography for the detection of acute coronary syndrome in patients with acute chest pain: From the ROMICAT trial. Circ. Cardiovasc. Imaging 2010, 3, 375-383. [CrossRef]

35. Mahnken, A.H.; Bruners, P.; Katoh, M.; Wildberger, J.E.; Günther, R.W.; Buecker, A. Dynamic multi-section CT imaging in acute myocardial infarction: Preliminary animal experience. Eur. Radiol. 2006, 16, 746-752. [CrossRef] [PubMed]

36. Moore, A.; Goerne, H.; Rajiah, P.; Tanabe, Y.; Saboo, S.; Abbara, S. Acute Myocardial Infarct. Radiol. Clin. N. Am. 2019, 57, 45-55. [CrossRef]

37. Kalra, P.R.; Ohri, S.K.; Morgan, J.M. Images in cardiology. Mitral regurgitation secondary to ruptured papillary muscle. Heart 2000, 84, 13. [CrossRef] [PubMed]

38. Figueras, J.; Cortadellas, J.; Soler-Soler, J. Left ventricular free wall rupture: Clinical presentation and management. Heart 2000, 83, 499-504. [CrossRef] [PubMed]

39. Motoyama, S.; Ito, H.; Sarai, M.; Kondo, T.; Kawai, H.; Nagahara, Y.; Harigaya, H.; Kan, S.; Anno, H.; Takahashi, H.; et al. Plaque characterization by coronary computed tomography angiography and the likelihood of acute coronary events in mid-term follow-up. J. Am. Coll. Cardiol. 2015, 66, 337-346. [CrossRef] 
40. Higashigaito, K.; Hinzpeter, R.; Baumueller, S.; Benz, D.; Manka, R.; Keller, D.I.; Alkadhi, H.; Morsbach, F. Chest pain CT in the emergency department: Watch out for the myocardium. Eur. J. Radiol. Open 2018, 5, 202-208. [CrossRef]

41. Schlett, C.L.; Banerji, D.; Siegel, E.; Bamberg, F.; Lehman, S.J.; Ferencik, M.; Brady, T.J.; Nagurney, J.T.; Hoffmann, U.; Truong, Q.A.; et al. Prognostic value of CT angiography for major adverse cardiac events in patients with acute chest pain from the emergency department: 2-year outcomes of the ROMICAT trial. JACC Cardiovasc. Imaging 2011, 4, 481-491. [CrossRef] [PubMed]

42. Iannaccone, M.; Quadri, G.; Taha, S.; D’Ascenzo, F.; Montefusco, A.; Omede', P.; Jang, I.-K.; Niccoli, G.; Souteyrand, G.; Yundai, C.; et al. Prevalence and predictors of culprit plaque rupture at OCT in patients with coronary artery disease: A meta-analysis. Eur. Heart J. Cardiovasc. Imaging 2016, 17, 1128-1137. [CrossRef] [PubMed]

43. Chinnaiyan, K.M.; Safian, R.D.; Gallagher, M.L.; George, J.; Dixon, S.R.; Bilolikar, A.N.; Abbas, A.E.; Shoukfeh, M.; Brodsky, M.; Stewart, J.; et al. Clinical Use of CT-Derived Fractional Flow Reserve in the Emergency Department. JACC Cardiovasc. Imaging 2020, 13 Pt 1, 452-461. [CrossRef]

44. Andreini, D.; Modolo, R.; Katagiri, Y.; Mushtaq, S.; Sonck, J.; Collet, C.; Martini, S.D.; Roberto, M.; Tanaka, K.; Miyazaki, Y.; et al. Impact of Fractional Flow Reserve Derived From Coronary Computed Tomography Angiography on Heart Team Treatment Decision-Making in Patients with Multivessel Coronary Artery Disease: Insights From the SYNTAX III REVOLUTION Trial. Circ. Cardiovasc. Interv. 2019, 12, e007607. [CrossRef] [PubMed] 